\title{
EXTENDED HYBRID REGION GROWING SEgMentation of Point Clouds With DIFFERENT RESOLUTION FROM DENSE AERIAL IMAge MATCHING
}

\author{
Mohammad Omidalizarandi ${ }^{1}$ and Mohammad Saadatseresht ${ }^{2}$ \\ ${ }^{1}$ University of Stuttgart, Institute for Photogrammetry, Geschwister-Scholl-Str. \\ 24D, 70174, Stuttgart, Germany \\ mohammadzarandiegmail.com \\ ${ }^{2}$ University of Tehran, Faculty of Engineering, Department of Geomatics \\ Engineering, North Amirabad street, Tehran, Iran \\ msaadateut.ac.ir
}

\begin{abstract}
In the recent years, $3 D$ city reconstruction is one of the active researches in the field of photogrammetry. The goal of this work is to improve and extend region growing based segmentation in the $X-Y-Z$ image in the form of $3 D$ structured data with combination of spectral information of $R G B$ and grayscale image to extract building roofs, streets and vegetation. In order to process $3 D$ point clouds, hybrid segmentation is carried out in both object space and image space. Our experiments on two case studies verify that updating plane parameters and robust least squares plane fitting improves the results of building extraction especially in case of low accurate point clouds. In addition, region growing in image space has been derived to the fact that grayscale image is more flexible than $R G B$ image and results in more realistic building roofs.
\end{abstract}

\section{KEYWORDS}

Object surface segmentation, Image segmentation, Region growing, $X-Y-Z$ image, Intensity

\section{INTRODUCTION}

In the current state-of-the-art, integrating the images with 3D data like range images incorporates computer vision and photogrammetry in this area of research. Automatic 3D reconstruction from $3 \mathrm{D}$ point clouds or range image is still one of the active research areas that has many applications in forestry, urban planning, tourist information systems and so on.

3D data measurements are grouped with respect to their similarity measures to define meaningful, coherent and connected segments. Acquisition of the dense and high accurate 3D data can assist us in the direction of automatic extraction of building models. Image matching can be considered a renaissance in the modern photogrammetry due to generating dense and high accurate point clouds with low price in comparison with LiDAR data. Building extraction is still challenging issue due to the complex building roofs, occlusions and shadows. Availability of the 3D structured data for each pixel and intensity values from the high resolution aerial images in addition to usage of high performance computer can assist us to deal with the challenging issues

David C. Wyld (Eds) : ICCSEA, SPPR, CSIA, WimoA - 2013

pp. 125-134, 2013. @ CS \& IT-CSCP 2013

DOI : $10.5121 /$ csit.2013.3513 
and achieving better segmentation results with low price. Thus, this combination can be considered the important step to reach the goal of automatic 3D reconstruction and object recognition.

Methods of building extraction can be categorized in two main groups of model-driven and datadriven. In model-driven approaches, firstly, building parameters are estimated and thereof these set of parameters are investigated in a model library for an appropriate model among primitive building models [1]. In data-driven approaches, point clouds can be segmented on the basis of proximity of the point clouds or similarity measures like locally estimated surface normals [2]. In the context of data-driven approaches, point cloud segmentation is usually carried out by surface growing, 3D Hough-transform and RANSAC [3]. Our focus in this paper is based on regiongrowing as the most prevailing method in building roofs extraction.

The goal of this work is to improve and extend region growing based segmentation in the $\mathrm{X}-\mathrm{Y}-\mathrm{Z}$ image in the form of 3D structured data with combination of spectral information of RGB and grayscale image to extract building roofs, streets and vegetation.

Building roofs extraction is the most important feature in our segmentation procedure. But, in some cases, there is an overlap between the building roofs and trees. Thus, it enforces us to classify trees and building roofs to get rid of this problem. In this work, vegetation is extracted by means of spectral information from RGB image. Generally speaking, in RGB image, vegetation is illustrated by the green color. However, this green color is not the same in all pixels of the RGB image. Therefore, we consider the threshold for the green channel of the RGB image and considering those pixels as vegetation which the differences of two other channels from green channel are above the pre-defined threshold. Filtering the extracted vegetation eliminate noisy pixels from the extracted vegetation and fill the gaps between them. In this work, we applied median filter with mask size 14 to remove the noises from the image and achieve better result of building and street extraction. Finally, morphologic dilation can be utilized to fill the gaps between extracted vegetation.

\section{RELATED WORKS}

Surface growing in object space is the equivalent of region growing in image space. Surface growing in object space can be performed by grouping point clouds which are spatially close and share similar measure properties like the direction of a locally estimated surface normal, gradient and the principal curvatures. As a result of surface growing method, point clouds are segmented into multiple surfaces. Surface growing algorithm starts from the optimal seed points and surfaces extend to neighboring point clouds based on pre-defined criteria.

As a privilege of surface based algorithms in comparison with edge based algorithm is no need to identify the surface boundaries at the preliminary step. Due to their easy implementation and well time performance, surface growing algorithms are the prevailing method for point cloud segmentation. However, the weakness of this approach is connected with a proper choice of the seed points. Furthermore, selecting different seed points may results different segmentation regions [4]. In addition, this algorithm tends to generate distorted boundaries due to segment objects in the region level instead of pixel level [5].

Jarząbek [6] proposed segmentation algorithm based on updating plane parameters and merging surface patches which works well in case of high accurate point clouds. Therefore, we improved the aforementioned algorithm by considering robust estimation and removing outliers. In addition, no need of merging surface patches due to considering unlimited nearest neighboring points. However, it is quite time consuming and can be used merely for building roofs extraction. 
Rabbani et al. [7] proposed segmentation of point clouds using smoothness constraint. This approach worked well for the high accurate point clouds. However, it seems problematic in case of low accurate point clouds. Therefore, in this work, it has been implemented to segment streets to increase the run time of the implemented program and preventing updating the plane parameters.

Kada et al. [8] proposed sub-surface growing to extract complex building roofs as well. In this approach, model-driven and data driven are combined to gain the advantage of both methods. Firstly, surface growing is applied and virtual points are defined below the real points at subsurface level which allow surfaces grown larger and results in better estimation of plane parameters in company of better estimation of shape primitive model parameters.

\section{The Proposed Extended Region Growing-based Segmentation}

In this work, segmentation method is applied based on region growing. This approach has been applied in both object space and image space. The first step in region growing is to select a set of seed points. The initial region starts from optimal seed points. Then, regions are grown from these seed points to neighboring points depending on a similarities criterion. The criterion in object space is the orthogonal distances of the 3D point clouds from the plane and angle threshold between surface normal of the seed point and its neighborhood. In addition, the criterion in image space can be pixel intensity. Seed point selection is the important step and final segmentation results are dependent on it. In order to find optimal seed point, firstly, plane equation is defined for each seed point and its neighboring point clouds. Then, the residuals (orthogonal distances of the point clouds to the best fitted plane) are computed. The point within the fitted plane with lowest square sum of residuals is considered the optimal seed point. Outliers would affect on the results of surfaces normal and consequently on the square sum of residuals which leads to a failure of detecting proper seed points of surfaces. Therefore, robust least squares adjustment is applied to detect the optimal seed point even in the presence of outliers. In this approach, firstly, the plane fit to the surface points and their neighborhood. The candidate point that its orthogonal distance to the fitted plane is below the pre-defined threshold is accepted as a new surface point. Due to increase the efficiency of the program while using low accurate point clouds, the plane equation is updated after adding the new candidate point to the corresponding surface points. The neighborhood threshold and residual threshold are used to determine the smoothness of the fitted plane. Secondly, the local surface normal at each point is compared with its neighboring points. The neighboring points are accepted if the angle between its surface normal and normal at the neighboring point is below the pre-defined threshold.

The segmentation method has two steps, Surface normal estimation and region growing that is described and discussed in next sections.

\subsection{Robust Plane Fitting}

The least squares fitting approach can result to optimal plane fitting parameters while there are no outliers in the datasets. This approach may lead to failure in case of noisy points or isolated points that are far from the plane. Based on the residuals (orthogonal distances of 3D point clouds to the fitted plane) from the previous adjustment, Danish robust estimation gives each observation a suitable weight using equation (1). In this work, at the first, surface normal is calculated using least squares fitting (LSF). Then orthogonal distances of 3D point clouds to the fitted plane are calculated. Note that normal vectors are already normalized and norm of them are equal to 1 . In the next step, standard deviation of calculated orthogonal distances from previous step is calculated. If an orthogonal distance is within 2 times of the calculated standard deviation, the point will be given a weight one. Otherwise, it will be given a weight zero to discard from our 
calculation. In the next step, weights are multiplied by 3D point clouds and surfaces normal are recalculated again to get better results.

$$
w_{i}= \begin{cases}1 & \left|d_{i}\right| \leq 2 \sigma \\ 0 & \left|d_{i}\right|>2 \sigma\end{cases}
$$

Where $w_{i}$ is weight of each 3D point cloud, $d_{i}$ is an orthogonal distance of 3D point clouds to the fitted plane and $\sigma$ is standard deviation.

\subsection{Algorithms of Region Growing in Object Space and Image Space}

The algorithms use the adjacency relations in the $\mathrm{X}-\mathrm{Y}-\mathrm{Z}$ image where $\mathrm{X}, \mathrm{Y}$ and $\mathrm{Z}$ values are available for each pixel. In order to implement region growing in object space, we use point normals and their residuals which already assigned to each image pixel. In addition, implementing region growing in image space can be carried out by using intensity values of image pixels. This algorithm in object space aims to group points which locally connected and belong to smooth surface. Furthermore, in image space, it aims to group image pixels with nearly similar intensity values. The points in a segment should locally make a smooth surface, whose normals do not vary too much from each other. In this procedure, angle threshold $\left(\theta_{t h}\right)$ between the selected seed point and its neighboring points is applied to add qualified points to the region. In the next step, using residual threshold $\left(r_{t h}\right)$ can lead to detect smooth surfaces in different height level [9].

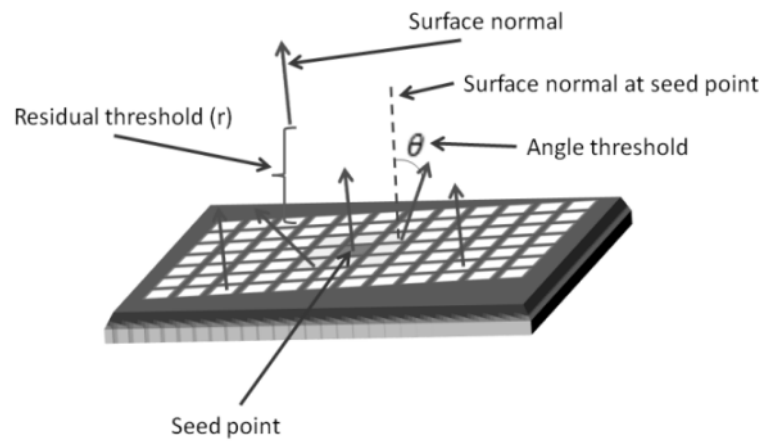

Figure 1. Representation of surface normals and segmentation parameters in object space

In this work, in order to calculate surface normals using robust estimation, the segmentation has been implemented in two different methods.

First method can be used to extract objects (building roofs and streets) for dense and high accurate point clouds. In this method, selected seed point is compared with its neighboring points with considering angle and residual threshold. In the next step, one of neighboring point can be considered as a seed point and this procedure is repeating. First approach leads to quite good results due to use dense and high accurate point clouds. This method, even can work to extract streets for low accurate point clouds because the surface normals in the flat areas do not vary too much. Extracting street by using this method can improve the run time of the program. 


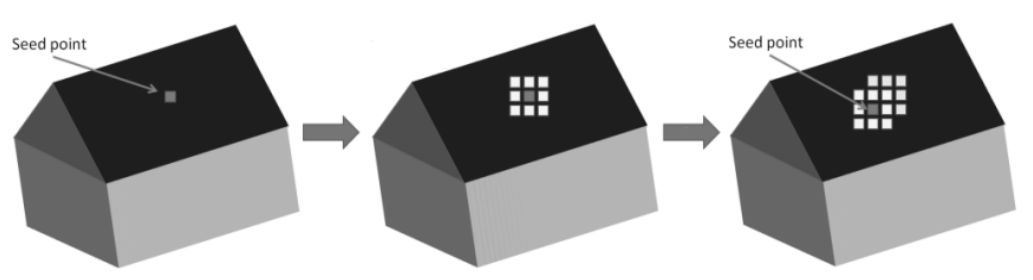

Figure 2. Representation of seed point and its neighboring points in first method

Second method can be used to extract building roofs for low accurate point clouds. In this method, optimal seed point is selected and it is compared with its neighboring points with considering angle and residual threshold. In the next step, those neighboring points that are qualified are added to surface points. Then, plane is updated and new plane parameters are calculated. Updating the plane parameters, can increase the efficiency of the algorithm in comparison with the first method. In this approach, we need to consider more neighboring points in comparison with the first approach due to use low accurate point clouds. Consequently, updating the plane parameter and considering more neighboring points can increase the run time of the program.

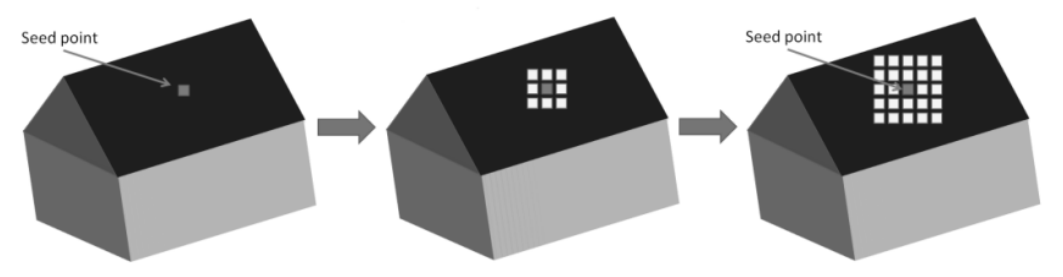

Figure 3. Representation of seed point and its neighboring points in second method

These approaches are investigated in different case studies by using RGB image, grayscale image and both of them. Using RGB image leads to extract building roofs with more detail but, the shape of extracted building roofs are not close to their realistic shape. On the other hand, using grayscale image results in more realistic building roofs and approximately can cover the whole building roofs due to more flexibility of gray values in region growing procedure. Segmentation in RGB image can be carried out by comparing three channels of the pixels in the image. Furthermore, it can assist us to detect vegetation from images based on comparing three channels and considering those pixels as vegetation which the differences of two other channels from green channel is above the pre-defined threshold. For instance, in the Table 1, RGB values from the RGB image compares to gray values from the grayscale image.

Table 1. Example of RGB values vs. gray values in the image segmentation procedure

\begin{tabular}{|c|c|c|c|c|}
\hline & Blue & Green & Red & Gray value \\
\hline (a) & 94 & 97 & 88 & $\mathbf{9 4}$ \\
\hline (b) & 95 & 97 & 89 & $\mathbf{9 4}$ \\
\hline (c) & 97 & 96 & 90 & $\mathbf{9 4}$ \\
\hline
\end{tabular}

As can be seen from the Table 1, combination of different RGB values can generate the same gray value that demonstrates the higher flexibility of the grayscale image in region growing especially, in extraction of the building roofs. This flexibility leads to better segmentation of the building roofs in the image space. 
DTM is also used as additional information to recognize ground and non-ground 3D point clouds. It can be applied by considering the nDSM threshold and separate point clouds to two main categories of terrain surfaces and building roofs.

$$
\mathrm{nDSM}=\mathrm{DSM}-\mathrm{DTM}
$$

\section{EXPERIMENTS AND RESULTS}

In this work, different types of point clouds with different level of density and accuracy are investigated. Therefore, we carried out our experiments in two case studies.

In the first case Study, we dealt with the dense and high accurate point clouds in addition to grayscale image. In this case study, first method of computing surface normals using robust estimation has been applied. This method leads to reasonable results with dense and high accurate point clouds for the extraction of building roofs. Furthermore, it also works well in the flat areas like streets even with lower accuracy due to not varying the slope of surfaces. During the process of segmentation, there is trade-off between the parameters such as angle threshold $(\theta)$, residual threshold(r) and numbers of nearest neighboring point threshold. Furthermore, segment size threshold is considered to remove small segmented regions. These results indicate that inappropriate threshold can lead to over or under-segmentation.
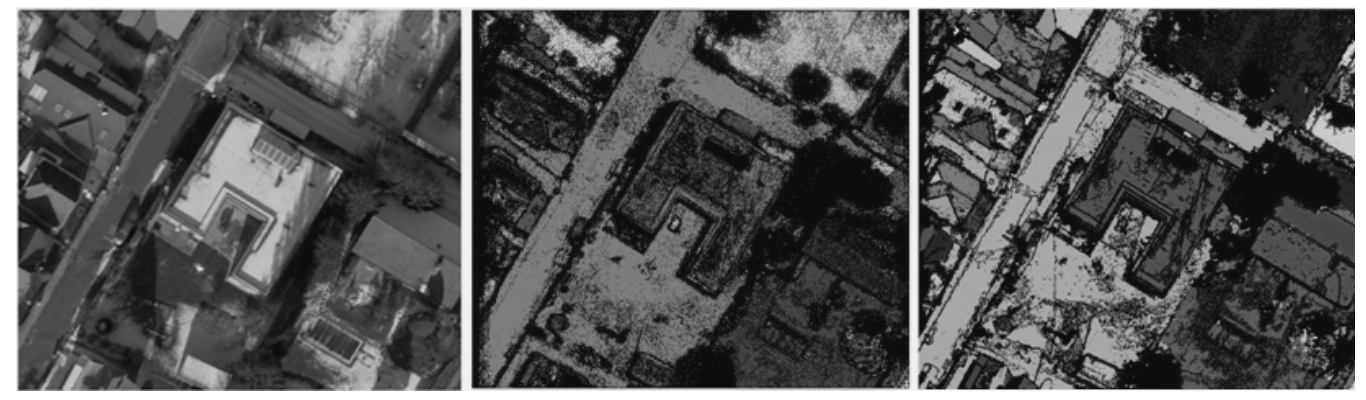

Figure 4. Base image (left), Segmentation of surface normals in object space using first algorithm (middle) and image space (right), $\theta<5^{\circ}, \mathrm{r}<0.1 \mathrm{~m}, 50$ nearest neighbours

In the second case study, three types of point clouds with different level of accuracy and density in company of RGB image and DTM have been utilized.

These three types of datasets are as follows:

- Sparse and high accurate point clouds

- Medium and less accurate point clouds

- Dense and low accurate point clouds 

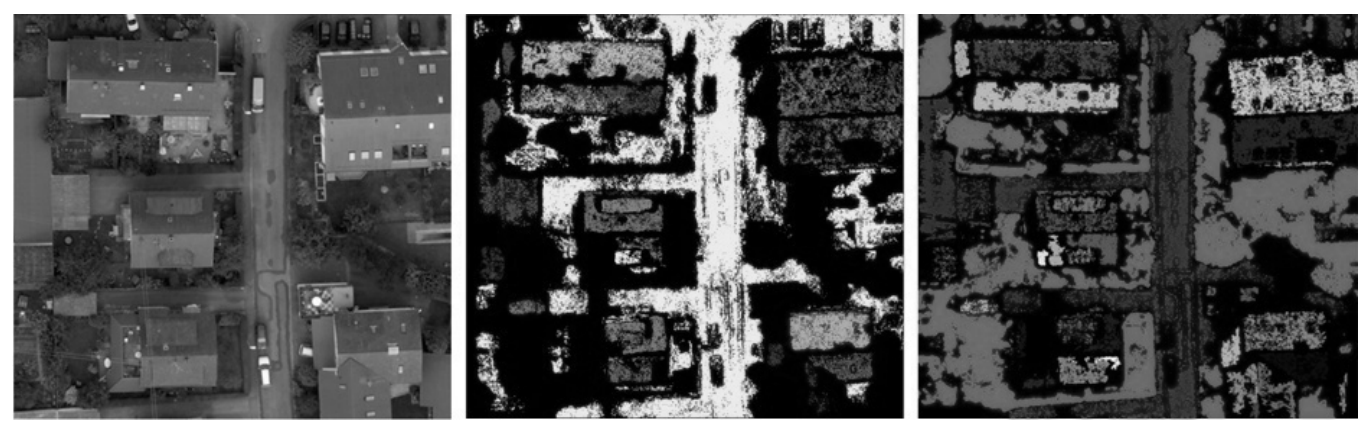

Figure 5. Base image (left), Segmentation of surface normals in object space using second algorithm (middle) and image space (right), $\theta<11.5^{\circ}, \mathrm{r}<0.4 \mathrm{~m}, 250$ nearest neighbors, nDSM threshold $=1.5$ [m]

First type of these data sets could not be processed individually because of lack of enough 3D point clouds to define surface normals. However, medium and dense 3D point clouds are processed separately. Furthermore, in order to improve the results of segmentation, these three types of data sets are combined together and results in generating forth type of data sets that is so called hybrid point clouds. In this case study, segmentation procedure like as case study I is performed in object space and image space. However, computation of surface normals is modified by updating planar surfaces that is grown from optimal seed points.

In this work, 3D point clouds were available in two file formats: LAS and txt file format. LAS format is the popular format to read and write point clouds. This file format can be read in C++ by the usage of LASreader class that belongs to LAStools. OpenCV is the proper library to work with the images and matrices with powerful functional operation. Furthermore, it has been used to solve eigenvalue problem and delineation of building roofs boundaries using convex hull. In addition, CGAL library provides us this capability to delineate building roofs boundaries using 2D $\alpha$-shape. Segmented 3D point clouds are stored in VRML format to display them in 3D and enables users to walk or fly through the segmented regions.

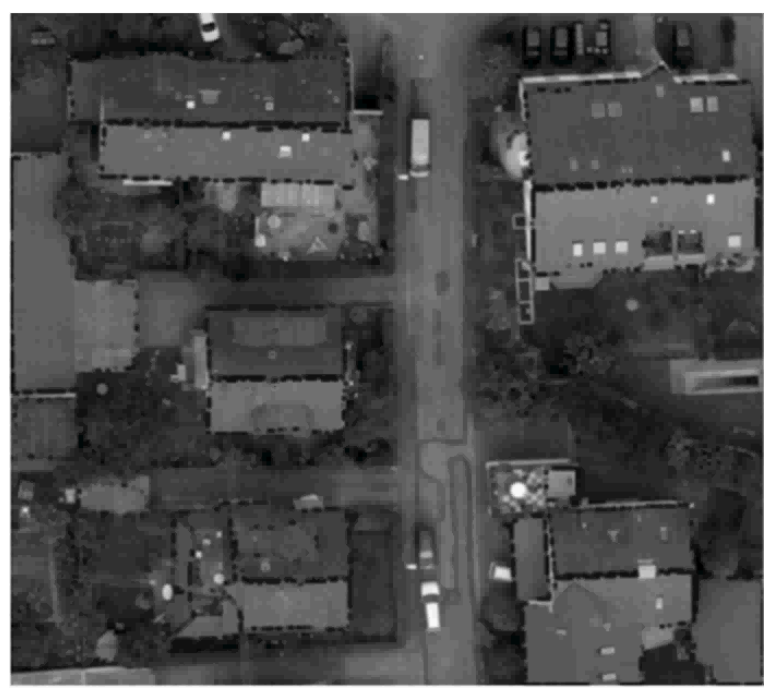

Figure 6. Representation of surface boundaries with points using 2D alpha shapes 
Table 2 represents the evaluation of the validated 3D point clouds in object space. It is carried out by computation of the standard deviation and mean $\mathrm{Z}$ shift with respect to the orthogonal distance between the 3D point clouds and the best fitted plane in extracted building roofs and flat terrain.

Table 2. Validation of hybrid point clouds

\begin{tabular}{|c|c|c|}
\hline Surface type & Building roofs & Flat terrain \\
\hline Standard deviation of validated points & $0.409 \mathrm{~m}$ & $0.473 \mathrm{~m}$ \\
\hline Mean Z shift of validated points & 0.0 & 0.0 \\
\hline
\end{tabular}

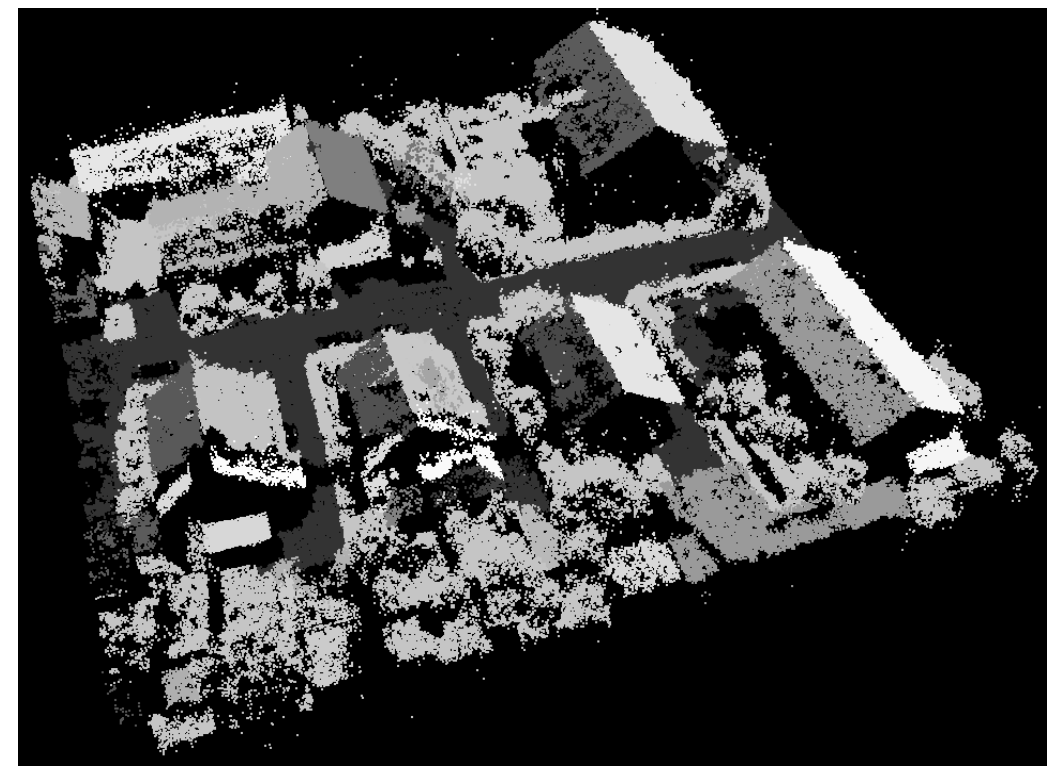

Figure 7. 3D visualization of segmented 3D point clouds in VRML format

\section{Conclusions}

Robust estimation has been improved the result of surface normals from least squares plane fitting by eliminating the effects of the noises and outliers. However, it also increased the runtime of the program with recalculating the surface normals.

First method of segmenting surface normals worked well for the dense and accurate point clouds and furthermore, was utilized to extract streets due to not varying the surface normals in the flat areas. The second method was carried out for the extraction of building roofs and worked well in most cases and especially applicable in case of low accurate point clouds. Second method was preferred to the first method in the extraction of building roofs due to updating the plane parameters and derived better result.

Availability of the 3D coordinates for each image pixel in the form of X-Y-Z image in addition to intensity values from RGB image or grayscale image can assist us to better interpret and recognize the objects and it can be considered the good combination in order to reach the goal of automatic $3 \mathrm{D}$ city reconstruction and object recognition. 
Vegetation extraction has been led to failure in some image pixels that their intensity values differed too much from their realistic color in the nature. In addition, combination of $\mathrm{X}-\mathrm{Y}-\mathrm{Z}$ image with RGB image results in extraction of building roofs with more detail due to comparing three channels of the RGB image together and therefore makes it less flexible in region growing procedure in image space. Hence, the extracted building roofs boundaries from RGB image was not as good as building roofs boundaries from grayscale image.

Delineation of building roofs boundaries using 2D alpha shapes, result in better and realistic building roofs boundaries with more detail. However, it is performed by installing the huge library that increases the run-time of the program. Furthermore, there is trade-off between alpha values to choose the appropriate value.

The novelty of this work comparing to previous work is proceeding of surface growing basedapproach with unlimited neighboring points and thereof no need of merging surface patches. In addition, robustness of the computation of surface normals assists us to discard outliers. Furthermore, segmentation in object space is applied in two different methods to increase the run time of the program in the procedure of extracting building roofs and streets. Furthermore, segmentation was investigated in two case studies to observe the effect of low accurate point clouds in the procedure of object recognition. In addition, RGB image compared to grayscale image in the process of segmentation in the image space to obtain better results.

As a recommendation for the future work, performing the segmentation procedure in object space and image space simultaneously by the usage of 3D point clouds in the form of X-Y-Z image in company of gray value from grayscale image may lead to better results of the segmentation and reach closer to the goal of automatic 3D city modeling and object recognition. In addition, performing segmentation in object space and thereafter in image space was also successful. However, it will derive better results by computing intersection of the adjacent building roofs for those building roofs that merged due to having similar intensity values. It is carried out by the usage of surface normals of the best fitted plane for each building roof and finding intersection lines between them.

\section{REFERENCES}

[1] Maas, H.-G. and Vosselman, G., (1999) "Two Algorithms for Extracting Building Models from Raw Laser Altimetry Data", ISPRS Journal of Photogrammetry \& Remote Sensing Vol. 54, No. 2/3,pp 153-163.

[2] Vosselman, G., Gorte, B., Sithole, G., Rabbani, T., (2004) "Recognizing Structure in Laser Scanner Point Clouds", International Archives of Photogrammetry, Remote Sensing and Spatial Information Sciences, 46(part 8/W2) 33-38.

[3] Vosselman, G., (2010) "Visualization and Structuring of Point Clouds", In: George Vosselman, HansGerd Maas (eds.) Airborne and Terrestrial Laser Scanning, Whittles Publishing, pp 45-81.

[4] Biosca, J.M., Lerma, J.L., (2008) "Unsupervised robust planar segmentation of terrestrial laser scanner point cloud based on fuzzy clustering methods", ISPRS Journal of Photogrammetry and Remote Sensing, 63, pp 84-98.

[5] Bab-Hadiashar, A. and Gheissari, N., (2006) "Range image segmentation using surface selection criterion", IEEE transactions on image processing, Vol. 15, No. 7.

[6] Jarząbek, M., (2009) "Segmentation and presentation of multiple 3D point clouds", Master thesis, University of Stuttgart, Germany.

[7] Rabbani, T., Heuvel, F.A. van den, Vosselman, G., (2006) "Segmentation of point clouds using smoothness constraint", International Archives of the Photogrammetry, Remote Sensing and Spatial Information Sciences, 36(Part 5) 248-253.

[8] Kada, M., Wichmann, A., (2012) "Sub-surface growing and boundary generalization for 3D building reconstruction", ISPRS Annals of the Photogrammetry, Remote Sensing and Spatial Information Sciences, Volume I-3, Melbourne, Australia. 
[9] Rabbani, T., (2006) "Automatic reconstruction of industrial installations using point clouds and images", Publications on Geodesy, 62, Delft, The Netherlands.

\section{Authors}

Mohammad Omidalizarandi received a B.Sc. degree (Surveying Engineering) from the Iran University of Science and Technology (2004) and M.Sc. degree (Geomatics Engineering) from the University of Stuttgart (2011). He taught Photogrammetry, Image Processing and Geodesy for undergraduate students in Golestan University. His current research interests include Building reconstruction, Panorama, Image Matching and Videogrammetry.

Mohammad Saadatseresht received a B.Sc. degree (Surveying Engineering) (1996), M.Sc. degree (Photogrammetry) (1998) and PhD (Close range Photogrammetry) (2004) from Tehran University. He teaches Industrial Photogrammetry, Close range Photogrammetry, Photogrammetry and Image Processing for undergraduate, M.Sc. and $\mathrm{PhD}$ level Students. He is currently at the University of Tehran and working as associate professor.
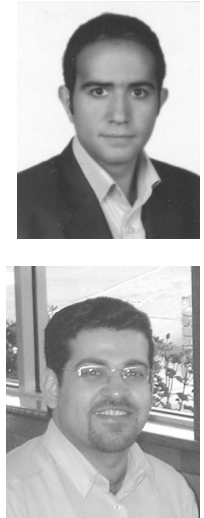